\title{
La Accesibilidad a la Administración Electrónica en España de las Personas con Discapacidad Motora
}

\section{Accessibility to the Electronic Administration in Spain of \\ Persons with Motor Disability}

Marta Aguilar Gil

Doutorado em Sociologia pela Universidad Pontificia de Salamanca. Professora de Sociologia pela Universidad de Sevilla, Espanha. maguilar6@us.es

https://orcid.org/0000-0002-8038-3421

Rafael Conde Melguizo

Doutorado em Sociologia pela Universidade de Sevilla. Professor de Sociologia, Marketing y Diseño Universal da Escuela Universitaria de Diseño, Innovación y Tecnología, Universidad Camilo José Cela, Espanha.

rafaelcondemelguizo@gmail.com

https://es.linkedin.com/in/rafaelcondemelguizo

Resumen: La Ley 11/2007 de Acceso Electrónico de los Ciudadanos a los Servicios Públicos (Boletín Oficial del Estado 2007) establece la obligación de que los ciudadanos españoles puedan realizar todas sus gestiones con la administración pública de forma electrónica en igualdad de condiciones de acceso a los servicios. En este trabajo se identifican las barreras que impiden a las personas con lesión medular y parálisis cerebral acceder a los servicios de la administración pública electrónica de manera autónoma y eficaz. Se aportan evidencias de que las soluciones de accesibilidad y/o diseño universal no son suficientes para permitir su acceso a la administración en igualdad de condiciones que el resto de ciudadanos debido a la presencia de elementos de naturaleza sociológica, como el autoestigma o las formas de socialización en la discapacidad, que condicionan su relación/interacción con los elementos técnicos. Hemos llegado a la conclusión de que el paradigma técnico-funcional resulta insuficiente para comprender todos los procesos que pueden observarse en un estudio de accesibilidad. Se hace imprescindible la aplicación de la sociología para el análisis de variables determinantes para la accesibilidad a las nuevas tecnologías por parte de personas con discapacidad. La socialización primaria es el elemento sociológico que se ha revelado más influyente en las capacidades de las personas con discapacidad. Dentro de las posibilidades de socialización, el estigma aparece de manera más determinante en las personas que viven la discapacidad desde el nacimiento que en aquellas cuya discapacidad es sobrevenida. La conversión del estigma en autoestigma discapacita a la persona en capacidades para las que funcionalmente no está discapacitada. Esta conclusión invita a tomar en cuenta a la sociología como una ciencia útil y necesaria en los estudios de accesibilidad, hoy limitados a los campos técnicos de la ingeniería y el diseño.

Palabras clave: estigma; diseño universal; servicios publicos; paralisis cerebral; lesión medular.

Abstract: Spanish law 11/2007, on Electronic Access of Citizens to Public Services (LAECSP) (BOE, 2007), establishes the obligation to guarantee that all Spanish citizens have the means to carry out all transactions with public administration electronically under conditions of equal access. In this article, we identify the barriers to persons with spinal cord injury and cerebral paralysis. We provide evidence on how accessibility solutions and/or universal design are not enough to permit their access to eGovernment on an equal footing with other citizens. Public policies designed to implement Act $11 / 2007$ on Electronic Access to Public Services by citizens, LAECSP (BOE, 2007) must include social interventions with these groups, in addition to work on technology. We have reached the conclusion the techno-functional paradigm is not sufficient to understand all of the processes that can be observed in the study of accessibility. Sociological research is essential to analyse determinants of accessibility to new technologies for persons with disability. Primary socialization is the sociological factor that has the greatest impact on the capacities of persons with disabilities. Within the processes of socialization, stigma appears to be more of a determinant in persons who have lived with disability since birth than in those with acquired disability. The conversion of stigma into self-stigma discapacitates individuals, even for tasks in which they face no functional discapacity. This conclusion invites to consider sociology as a science useful and necessary in studies of accessibility, now limited to the technical fields of engineering and design.

Key words: stigma; Universal design; public services; cerebral palsy; spinal cord injury

Texto completo em português: http://www.apgs.ufv.br Full text in Portuguese: http://www.apgs.ufv.br

\section{INTRODUCCIÓN}

La problemática que ha abordado el siguiente trabajo ha sido la necesidad de identificar y comprender los problemas de accesibilidad de las personas con discapacidad motora a la administración pública electrónica desde un punto de vista sociológico, con el fin de promover su participación ciudadana en igualdad de condiciones, pudiéndose beneficiar de las oportunidades de la e-Administración y la sociedad de la información.

A lo largo de la experiencia profesional de los autores, al abordar estudios de accesibilidad se ha observado que surgen elementos de naturaleza sociológica que condicionan la relación/interacción de las personas con discapacidad con la tecnología. Estos elementos no son explicables con las herramientas de diseño accesible o universal actuales, propias de las ciencias técnicas, la ingeniería y el diseño, sino que sólo son comprensibles con la aplicación de la sociología.

Existe bibliografía de referencia sobre la accesibilidad de carácter técnico (Nielsen, 1994; Henry, 2001; Hassan, 2001, 2004) e incluso de carácter multidisciplinar (Granollers y Saltiveri, 2009; Valero, 2011), incluyendo algunos elementos de las ciencias sociales. Del mismo modo existe extensa bibliografía de referencia que podemos denominar como sociología de la discapacidad (Finkelstein, 1980; Barton, 1998, p.19-33; Romañach, 2005; Etxeberría, 2008; Ferreira, 2006, 2008, 2009, 2010; Palacios, 2006, 2007; Toboso, 2007, 2010; Díaz Velázquez, E., 2010; Ferrante, 2010; Maraña, 2010, p.259-281;

Correspondência/Correspondence: Marta Aguilar Gil. Universidade de Sevilha. Calle San Fernando, 4, 41004 Sevilla, Espanha. maguilar6@us.es 
Rojero, 2010). Sin embargo, existe un gap entre ambos conocimientos. Es necesario por tanto crear un espacio donde ambos conocimientos confluyan y donde las aportaciones de la sociología de la discapacidad ayuden a la comprensión de los fenómenos observados en los trabajos de accesibilidad de difícil resolución por métodos únicamente técnicos. Este es el espacio que hemos pretendido abrir en esta investigación.

El objetivo principal de esta investigación ha sido ofrecer resultados científicamente relevantes que permitan reivindicar la sociología como una ciencia útil y necesaria en los estudios y trabajos prácticos de diseño de accesibilidad a la tecnología. Los resultados nos permiten consolidar nuestra hipótesis y, por tanto, realizar esta reivindicación.

Este artículo refleja los resultados de una investigación sociológica realizada en el seno del proyecto ASTIC "Accesibilidad en los Servicios Telemáticos Inteligentes para el Ciudadano", coordinado por la Federación Nacional ASPAYM (Asociación Nacional de Parapléjicos y Grandes Discapacitados Físicos) y financiado por el "Plan Avanza: Ciudadanía Digital del Ministerio de Industria, Turismo y Comercio". Dentro del consorcio de investigación han participado las siguientes entidades: el departamento de Ingeniería y Arquitecturas Telemáticas (DIATEL) de la Universidad Politécnica de Madrid (UPM), la Fundación Vodafone España y la Asociación de Padres de Alumnos Minusválidos (APAM).

\section{FUNDAMENTOS TEÓRICOS}

\subsection{Modelo biopsicosocial de la discapacidad}

La investigación se ha fundamentado en comprender la discapacidad desde el paradigma de consenso internacional conocido como el Modelo Biopsicosocial establecido por la Organización Mundial de la Salud en la "Clasificación Internacional de Funcionamiento, Discapacidad y Salud" -en adelante CIF- en 2001 (World Health Organization, 2001). Según el modelo biopsicosocial, la discapacidad es un fenómeno que no puede comprenderse sólo desde la perspectiva médica o la perspectiva social, sino mediante una combinación de ambos modelos. De esta manera, se establece un ecosistema de elementos que influyen en el individuo. Estos elementos son:

Deficiencias. El CIF entiende una deficiencia como una desviación de la norma generalmente aceptada en relación al estado biomecánico del cuerpo y sus funciones (WHO, 2001, p. 12).

Factores contextuales. Son aquellos que explican el contexto en el que la deficiencia tiene lugar. EI CIF distingue entre factores ambientales y factores personales.

O Los factores ambientales incluyen el ambiente físico, social y actitudinal en que las personas viven y desarrollan sus vidas. Los factores ambientales pueden tener una influencia positiva o negativa sobre la deficiencia identificada (WHO, 2001, p. 16).

- Se refieren a las características de un individuo que no forman parte de una condición de salud: género, etnia, edad, educación, profesión, etc. Estos elementos pueden desempeñar un papel en la discapacidad (WHO, 2001, p.17)
Restricciones a la actividad y/o la participación. La discapacidad desde el modelo biopsicosocial puede ser interpretada como un proceso de interacción de los diferentes elementos del modelo. Los conceptos clave para comprender esta interacción se definen en dos dicotomías (WHO, 2002, p.10-13):

O Actividad y Participación.

- Actividad es la ejecución de una tarea o acción por una persona. Por ejemplo, la capacidad de expresar sus propias ideas de forma comprensible.

- Participación es el desenvolvimiento de una persona en una situación vital. Por ejemplo, mantener una conversación.

○ Capacidad y Rendimiento.

- Capacidad describe la habilidad de un individuo para ejecutar una tarea -desplazarse en una silla de ruedas -.

- Rendimiento se refiere a lo que es capaz de hacer un individuo en su entorno habitual -imposibilidad de utilizar el transporte público, porque no está adaptado a personas en silla de ruedas-

Mediante este esquema, el modelo biopsicosocial sitúa en el mismo nivel de importancia los factores físicos del cuerpo de la persona y los factores ambientales, siendo la discapacidad el resultado de la relación entre ellos.

\subsection{Diseño Universal}

Tras la publicación del CIF y la generalización del modelo biopsicosocial como paradigma de consenso, se renovaron los conceptos destinados a definir las políticas para mejorar las condiciones de vida de las personas con discapacidad. Entre estos conceptos encontramos el de diseño universal.

El diseño universal fue definido en 2006 en la Convención sobre los Derechos de las Personas con Discapacidad y su Protocolo Facultativo como el "diseño de productos, entornos, programas y servicios que puedan utilizar todas las personas, en la mayor medida posible, sin necesidad de adaptación ni diseño especializado" (Organización de Naciones Unidas, 2006). Es necesario aclarar que cuando se habla de diseño universal no se habla únicamente de espacios físicos, sino también del entorno natural, ayudas, los espacios virtuales, espacios sociales, etc. El diseño universal se refiere a la creación desde su génesis de un entorno accesible que permita dotar a todos los individuos para ejercer su autonomía personal.

Partiendo de esta perspectiva, la pregunta de investigación planteada ha sido: “¿existen elementos sociales, propios del estudio por parte de la ciencia sociológica, que se revelen fundamentales a la hora de estudiar la accesibilidad a la tecnología por parte de las personas con discapacidad?" El objetivo principal de la investigación se ha definido como: "ofrecer información científicamente relevante sobre la importancia de la sociología para el estudio y comprensión de la accesibilidad a la tecnología”.

\subsection{Marco sociológico}

La perspectiva sociológica de la investigación ha partido del paradigma socio-técnico. (Bloor, 1991; Domènech y Tirado, 1998; Law y Hassard, 1999; Blanco, 2002; Latour, 2005; Sádaba y Gordo, 2008; 
Sánchez-Criado, 2008). Este paradigma defiende la interpretación de la relación de la tecnología y la sociedad como una misma realidad, en la que cada una de ellas es condición de posibilidad de la otra (Sádaba y Gordo, 2008). De este modo, existirán elementos sociales ajenos al diseño que influirán decisivamente en la relación e interacción de las personas con discapacidad motora con la administración electrónica, perpetuando la situación de exclusión y, por tanto, dificultando la igualdad de oportunidades en el acceso a los servicios.

La combinación del diseño para todos con la definición de funcionalidades del CIF ha sido denominada en esta investigación como paradigma técnico-funcional. Este paradigma busca definir desde el paradigma biopsicosocial las deficiencias y barreras del entorno, para posteriormente crear diseños universales que eviten las limitaciones en la actividad y la participación. Se trata, en resumen, de coordinar las funcionalidades con los apoyos técnicos para conseguir una accesibilidad universal.

Sin embargo, la inclusión de la perspectiva sociológica desvela carencias en la comprensión de la discapacidad desde el paradigma técnico-funcional. Para definir el marco teórico de la investigación que permitiese fundamentar la perspectiva sociológica y superar la visión del diseño universal, se llevó a cabo una exhaustiva revisión bibliográfica. Se han definido tres conceptos que amplían el paradigma de diseño universal: cuerpo normativo; distancia social; estigma. La elección y definición de estos conceptos surge de la revisión de la sociología de la discapacidad (Finkelstein, 1980; Barton, 1998:19-33; Maraña, 2003:259-281; Romañach, 2005; Palacios, 2006; Etxeberría, 2008; Ferrante, 2010; Ferreira, 2010; Díaz Velázquez, 2010; Rogero, 2010; Toboso 2010) y de la revisión de tradición sociológica de la sociología situacional (Goffman, 1961; 1979; 1981; Blumber, 1981; Ritzer, 1993; Joseph, 1999; Wolf, 2000; Díaz, 2002; Scambler, 2009; Marrero-Guillamón, 2012).

Frente al individualismo conceptual del paradigma técnicofuncional, la inclusión de la perspectiva sociológica exige la inclusión de los conceptos de cuerpo normativo y distancia social. El cuerpo normativo no es sólo lo normal, entendiendo como tal la mayoría estadística, sino que tiene un carácter de cuerpo normativamente impuesto. Existen determinadas capacidades asociadas a las necesidades definidas como básicas por nuestros patrones culturales de vida (ser productivos laboralmente, ser independientes en el desempeño de las actividades de la vida diaria, etc.) Del mismo modo, existen criterios normativos que especifican ciertas funciones corporales como las adecuadas para cubrir estas capacidades, llegando a confundirse funcionalidad con capacidad. El conjunto de capacidades y funcionalidades que se erigen por criterios sociales en normalidad, conforman el cuerpo normativo al que deben adaptarse todos los demás (Toboso, 2010, p. 76). La distancia social es el conflicto que se produce entre el cuerpo normativo y el cuerpo diverso -entendiendo este último como aquel que utiliza otras funcionalidades para alcanzar las capacidades establecidas socialmente como normales-.

El término diversidad funcional es un término acuñado en España en 2005 dentro del Foro de Vida Independiente para designar lo que habitualmente se conoce como discapacidad tratando de eliminar la negatividad en la definición y cambiarla por la idea positiva de diversidad (Romañach, 2005; Palacios, 2006: 28). La diversidad funcional reivindica una comprensión de la discapacidad dentro del modelo social. Para realizar una tarea, las diferentes personas pueden recurrir a diversas funcionalidades. Por ejemplo, para desplazarse por la ciudad se puede ir caminando, cojeando, sobre una silla de ruedas, etc. Existe por tanto una diversidad funcional para realizar una tarea, pero en el contexto social sólo alguna de ellas es considerada normal. Por ejemplo, caminar sería la funcionalidad normativa, siendo el resto de opciones catalogadas como inferiores (Palacios, 2006; Ferreira, 2010), aunque permitan el fin último, que es alcanzar la capacidad de desplazarse. De este modo, las personas que cuentan con una diversidad funcional -desplazarse en silla de ruedas-, son catalogadas como discapacitadas - no pueden andar, luego no tienen la capacidad de desplazarse-.

Es necesario hacer un inciso para aclarar que el camino de definición conceptual recorrido desde las primeras definiciones de la discapacidad en el campo de las ciencias de la salud hasta llegar a los conceptos de diversidad funcional y vida independiente que estamos manejando en este artículo permiten observar el crecimiento de la perspectiva social frente a la médica en el terreno teórico del mismo modo que queremos defender en esta investigación en el terreno empírico. Esta evolución puede consultarse en detalle en (Conde, 2014, p.155-175).

El mundo cotidiano está lleno de habilitadores tecnológicos que han sido diseñados para facilitar la capacidad de un ser humano promedio. Cuando estos habilitadores han sido diseñados para facilitar las capacidades mediante las funcionalidades propias del cuerpo normativo, pueden suponer una barrera para los cuerpos diversos. Por ejemplo, el diseño de unas escaleras para permitir la capacidad desplazarse mediante la funcionalidad normal de caminar. Sin embargo, las escaleras eliminan la capacidad de desplazarse mediante otras funcionalidades como ir sobre una silla de ruedas. Debido a que los habilitadores son de carácter artificial -escaleras- y a que su mediación es permanente, podemos afirmar que las capacidades -como subir dichas escaleras- son algo construido socialmente (Toboso, 2010, p.75). Desde este punto de vista, las barreras tecnológicas que encuentran las personas con discapacidad, son consecuencia de una distancia social entre su cuerpo diverso y el cuerpo normativo.

Pero aún existe un paso más en la definición de esta distancia: si se capacita a los cuerpos diversos mediante soluciones especializadas y adaptaciones su actividad no será valorada por el entorno social como una accesibilidad plena, puesto que sus funcionalidades no son equiparadas en eficacia con las propias del 
cuerpo normativo. Por ejemplo, si la persona en silla de ruedas es capaz de subir a un piso superior por una rampa, está capacitada para ascender como otra persona. Pero al no utilizar un habilitador pensado para el cuerpo normativo -escaleras -, sigue sin ser una funcionalidad legítima para ser considerado como capacitado. Esto implica que la distancia social se mantiene independientemente de que la barrera se haya superado desde el punto de vista del paradigma técnicofuncional. Cuando una persona utiliza una rampa construida desde la perspectiva del diseño universal está superando una barrera, pero está mostrando su condición de cuerpo diverso y, por tanto, experimentando una distancia social que le separa del resto y le estigmatiza.

\subsection{El estigma}

Según se ha explicado en el punto anterior, aunque la persona con discapacidad desarrolle las mismas capacidades que el cuerpo normativo gracias a adaptaciones del entorno, apoyos técnicos o la existencia de un contexto de diseño universal, al conseguirlo mediante otras funcionalidades y otros habilitadores, es observado por la sociedad como diferente al cuerpo normativo y, por tanto, aunque la distancia funcional se reduzca, la distancia social se mantiene.

El individuo será socializado en función de su diferencia con el cuerpo normativo. Recurriendo a la sociología situacional, cuando se produce este fenómeno hablamos de una socialización en el estigma (Goffman, 1961; 2006; Scambler, 2009; Aretio, 2010; Marrero-Guillamón, 2012)

El estigma tiene cuatro características fundamentales:

Se trata de una categorización desacreditante

Es una característica que desborda su origen y categoriza a todo el sujeto -sinecdoque-

Al ser totalizador, el estigma interviene en todas las interacciones del sujeto.

- La socialización en el estigma produce autoestigma. El sujeto se identifica con él (Goffman, 1961; 2006; Scambler, 2009).

Figura 1. Representación gráfica de las cuatro fases del estigma y su evolución desde la funcionalidad diversa hasta el autoestigma.

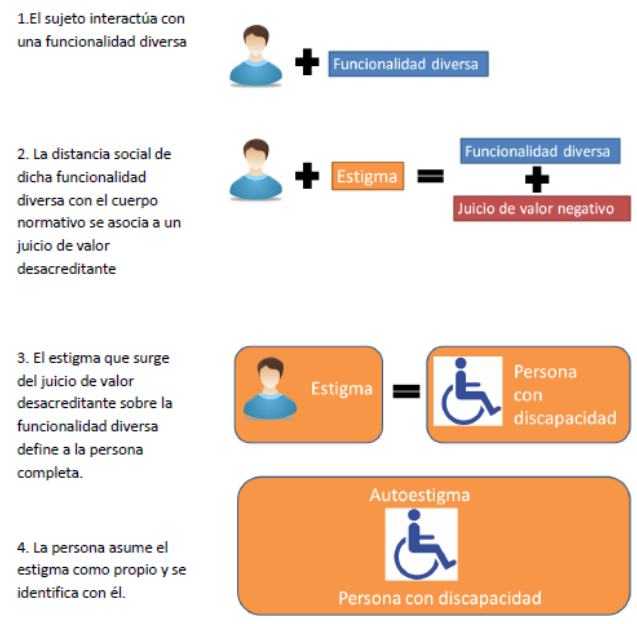

En resumen, el estigma tiene la característica de que no desacredita únicamente la funcionalidad distinta al cuerpo normativo, sino que desacredita a todo el sujeto. Esto hace que el estigma esté presente en todas sus interacciones y termine provocando la identificación del sujeto con el estigma mismo, en lo que se conoce como autoestigma. El autoestigma produce autolimitaciones del sujeto que exceden a las que se serían propias de su diversidad funcional.

Concretando este concepto a su aplicación práctica en la investigación realizada, estas autolimitaciones producidas por el autoestigma se manifiestan posteriormente en la accesibilidad a las tecnologías, produciendo problemas de accesibilidad que no son comprensibles desde el paradigma técnico-funcional, pero si desde la perspectiva sociológica.

\section{PROCEDIMIENTOS METODOLÓGICOS}

\subsection{Caso de estudio}

Para poner a prueba este marco teórico, se ha diseñado un estudio de caso con dos poblaciones de personas con discapacidad: personas con lesión medular y personas con parálisis cerebral. La elección de estas dos muestras responde a las diferencias en la socialización de las personas de ambas poblaciones.

O Por un lado, la lesión medular es una discapacidad sobrevenida. Las personas con lesión medular son sujetos que han tenido una socialización fuera de la discapacidad, pero debido a un accidente han visto mermadas funcionalidades motoras -paraplejia, tetraplejia, etc.- y han pasado a formar parte del colectivo de personas con discapacidad.

- Por el otro lado, la parálisis cerebral es una discapacidad que se adquiere en el nacimiento, por lo que se considera como una discapacidad congénita. Las personas con parálisis cerebral son sujetos que durante toda su vida han estado socializados como personas con discapacidad.

Con este planteamiento, la hipótesis de esta investigación ha quedado definida como: realizando las mismas pruebas de accesibilidad entre individuos de funcionalidades similares y con el uso de los mismos habilitadores tecnológicos, encontraremos diferencias en sus capacidades derivadas de sus diferencias de socialización, al menos igual de relevantes que las diferentes capacidades derivadas de sus diferencias funcionales.

La socialización en el estigma y el autoestigma limitará las capacidades del grupo con discapacidad congénita -parálisis 
cerebral- en mayor medida que aquellos cuya socialización en el estigma es sobrevenida -lesión medular-. Es necesario aclarar que la parálisis cerebral conlleva en ocasiones discapacidad cognitiva. Para la realización de esta investigación, las personas con parálisis cerebral participantes no eran afectadas por este tipo de discapacidad, sino que sufrían deficiencias físicas similares al colectivo de lesión medular.

Con todos los límites que plantea la metodología del estudio de caso (Sánchez Carrión, 1998; Coller, 2000; Yin, 2009), de confirmarse la hipótesis se confirmaría que las capacidades de las personas con discapacidad son limitadas y autolimitadas por su socialización en el estigma, reivindicando la sociología como una ciencia necesaria para las investigaciones sobre accesibilidad. A pesar de que nuestra investigación se limite a dos tipos de discapacidad motora, creemos que se trata de un planteamiento válido para el colectivo de personas con discapacidad que puede aplicarse en estudios similares con otras discapacidades, mediante la oportuna revisión de los indicadores utilizados en función de las características propias de cada colectivo.

La investigación se ha desarrollado en dos fases. La primera fase se ha centrado en el diseño de un escenario de pruebas dirigido a observar la interacción de las personas con discapacidad con la administración electrónica. La segunda fase ha desarrollado un análisis sociológico de los datos recogidos.

Para diseñar el escenario de pruebas, se procedió en primer lugar a la selección de un listado de servicios y una muestra representativa que permitiese la evaluación de la accesibilidad a los servicios de administración electrónica por parte del colectivo de personas con discapacidad motora. Se realizó una labor exhaustiva de documentación y se organizaron entrevistas grupales con personas con discapacidad motora y terapeutas ocupacionales de las asociaciones colaboradoras. Con la información recogida, se seleccionó una muestra estructural (Vallés M. S., 2003: 89-96) de 36 personas. Esta muestra se distribuía en 9 categorías determinadas por el cruce de dos variables que las personas con discapacidad identificaron como fundamentales en las entrevistas grupales:

O nivel de dependencia reconocido por la Ley de Promoción de la Autonomía de las Personas en situación de Dependencia (BOE, 2006): moderado, severo y gran dependiente. Los criterios de inclusión de los individuos en cada uno de los grupos son los propios definidos por la ley, ya que se solicitó a los participantes su calificación oficial por las administraciones públicas.

O nivel de manejo de las nuevas tecnologías: bajo, medio y alto. Para definir estos tres niveles, tanto en su número, como en su contenido, se realizaron entrevistas y pre-test con personas con discapacidad utilizando libremente un ordenador en las sedes de las asociaciones colaboradoras en la investigación.

Cada una de estas categorías recogía cuatro individuos para alcanzar el punto de saturación establecido en la bibliografía sobre pruebas de accesibilidad con usuarios (Nielsen, 1994; Henry, 2001; Hassan, 2004). Se procuró en la medida de lo posible que estos cuatro individuos fuesen dos hombres y dos mujeres para neutralizar la influencia que la variable género pudiese tener en el estudio y, de tenerla, poder identificar su importancia. Las personas fueron reclutadas por las entidades colaboradoras, realizándose pruebas en Madrid, Toledo, Cuenca, Albacete y Murcia. Cada individuo realizaba pruebas de accesibilidad con dos servicios, por lo que en total se han realizado 72 pruebas de accesibilidad con 36 personas sobre un total de 10 servicios seleccionados. Las pruebas tuvieron lugar en las sedes de las asociaciones colaboradoras. En estas pruebas se recogió información de tres fuentes:

O Un cuestionario técnico de accesibilidad construido ad hoc para la recogida de datos primarios. El cuestionario se denominó ACDM-16 y recogía en 16 preguntas los criterios WCAG 2.0 de accesibilidad referidos a la discapacidad motora (Conde, 2012)

- Una entrevista al usuario antes y después de la prueba para conocer su experiencia previa y sus expectativas futuras

- La observación directa del investigador

En las entrevistas grupales se definieron igualmente los 10 servicios básicos de la administración para la población observada.

Estos fueron:

Figura 2. Tabla de servicios seleccionados por los participantes en las entrevistas grupales.

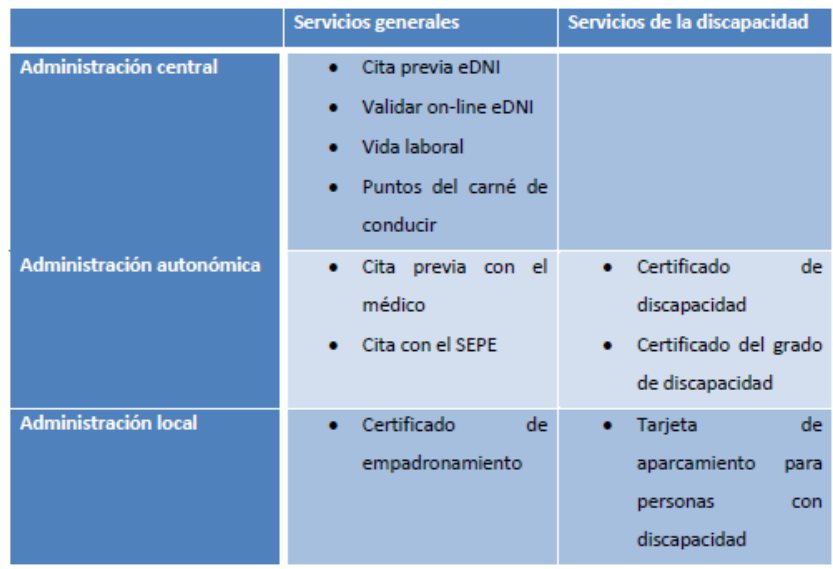

Cada usuario realizó dos gestiones con la administración, por lo que se realizaron en total 72 gestiones.

El análisis de los datos se centró en identificar las claves sociológicas de los resultados obtenidos en las pruebas piloto. En primer lugar, se realizó un análisis descriptivo de los datos recogidos en las pruebas, junto con una interpretación de los discursos recogidos en las entrevistas. Posteriormente, estos resultados se 
sometieron a deliberación en diversos grupos focales conformados por personas con discapacidad que habían participado en los pilotos.

En el análisis descriptivo se analizaron las 16 preguntas del cuestionario en relación a tres variables independientes: nivel de dependencia; experiencia previa en el uso de las nuevas tecnologías; discapacidad (parálisis cerebral o lesión medular).

\section{DISCUSIÓN Y ANÁLISIS DE DATOS}

Como resultado de este análisis se encontraron los siguientes resultados. La variable de nivel de dependencia se mostró irrelevante a la hora de explicar las posibles barreras identificadas por los individuos participantes en las pruebas. Del mismo modo, las capacidades funcionales tampoco mostraban influencia, pues individuos con capacidades similares tuvieron niveles de dificultad diferentes a la hora de acceder a los distintos servicios. Los apoyos técnicos utilizados tampoco ofrecían explicaciones lo suficientemente relevantes como para considerarse una variable explicativa. El nivel de manejo de las nuevas tecnologías si explicaba algunas barreras como la comprensión de los enlaces, la velocidad de navegación, etc., pero no era una variable capaz de explicar algunas barreras como la comprensión del texto o de las tareas que debían realizarse.

Sin embargo, al segmentar los resultados por tipo de discapacidad, aparecían diferencias relevantes, mostrando el colectivo con parálisis cerebral mayores dificultades que el colectivo con lesión medular a la hora de ejecutar las pruebas de accesibilidad. Se encontraron individuos con parálisis cerebral que eran capaces con pocos apoyos técnicos de completar tareas concretas, pero que manifestaban que no comprendían la gestión completa ni su utilidad. Por este motivo, manifestaban que, a pesar de las posibles soluciones técnicas del proyecto, no las realizarían en el futuro o que dichos servicios no eran útiles para ellos. Por el contrario, individuos con lesión medular que precisaron de mayores apoyos técnicos y un tiempo superior para realizar las mismas pruebas comprendían la gestión completa y manifestaban que era su intención realizar estas actividades autónomamente en el futuro.

Figura 3. Ejemplo de análisis descriptivo de los datos del cuestionario ACDM-16. Cruce de variable discapacidad con la pregunta "¿Comprende el texto?".

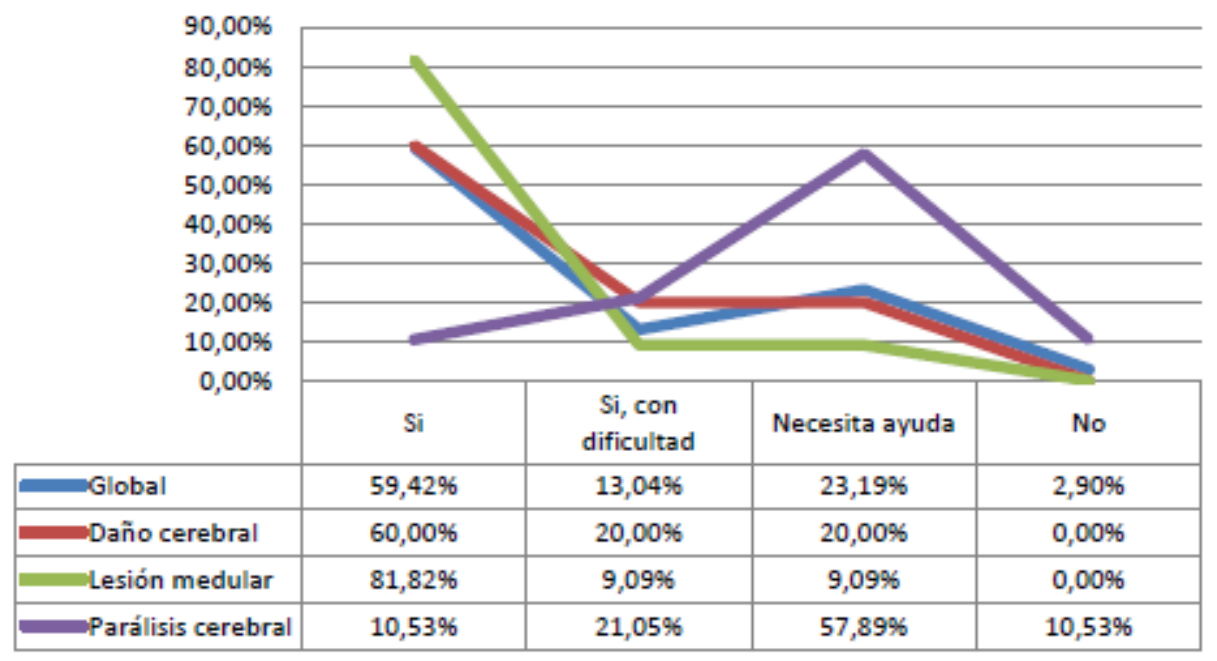

El análisis deliberativo se realizó en tres fases:

Un primer grupo de expertos (Campenhoudt, 2005; Dominguez, 2008, p. 99) conformado por profesionales de las asociaciones de la discapacidad colaboradoras. Concretamente, participaron una trabajadora social y una terapeuta ocupacional de cada una de las asociaciones de Madrid: ASPAYM Madrid y APAM. El objetivo de este grupo fue aprovechar la experiencia de los profesionales para diseñar los grupos focales deliberativos de personas con discapacidad.

En una segunda fase, se entrevistó a varias personas con discapacidad que había participado como usuarios en las pruebas. Concretamente, se entrevistó a una persona con lesión medular y dos personas con parálisis cerebral. El objetivo de las entrevistas fue adaptar la presentación de los datos descriptivos a un lenguaje que permitiese la deliberación dentro de los grupos.
Por último, se llevaron a cabo cuatro grupos focales deliberativos. Los grupos estaban formados por personas con discapacidad que habían participado en las pruebas piloto. Dos grupos en la sede de ASAPYM Madrid con personas con lesión medular y dos grupos en la sede de APAM con personas con parálisis cerebral. Cada grupo estaba compuesto por cuatro usuarios en las pruebas de accesibilidad, la persona entrevistada previamente y el investigador.

El grupo focal permite una mayor directividad que el grupo de discusión (Krueger, 1994; Greebaum, 1998; Dominguez, 2008, p.99). Para la moderación del debate grupo focal, se ha contado con un guión de tres puntos basado en las conclusiones del grupo de profesionales:

- Experiencia previa en las relaciones con la administración pública

○ Presentación de los datos descriptivos obtenidos. 
$\bigcirc \quad$ Análisis de la influencia del entorno en las barreras que hayan surgido en las pruebas de accesibilidad.

Los grupos tuvieron una duración aproximada de hora y media. Se grabaron en audio y se recogió información por parte del investigador en formato similar a un cuaderno de campo, apuntando frases literales, pero también actitudes, impresiones, comentarios, etc. que facilitan la comprensión de la información en el contexto en que se ha producido.

\section{RESULTADOS}

Los resultados de esta investigación reflejan que el estudio del estigma es un elemento necesario para la comprensión de la accesibilidad a las tecnologías. Debido a las diferencias de socialización entre los dos colectivos observados la presencia del estigma y el autoestigma ha sido distinta y fundamental para comprender las diferencias en la accesibilidad de ambos colectivos a la administración electrónica.

Los grupos focales han presentado dos colectivos diferentes con una presencia del estigma claramente distinta. El grupo de personas con parálisis cerebral se ha desvelado como un grupo muy homogéneo, con una identidad colectiva muy definida y una fuerte presencia del estigma y el autoestigma. Por el contrario, el grupo de personas con lesión medular ha resultado ser un grupo más heterogéneo, con mayor diversidad interna y una presencia del estigma diluida y variable. Las diferencias entre ambos grupos han resultado ser significativas a la hora de interpretar la accesibilidad a la administración electrónica y los datos recogidos en las pruebas piloto.

El colectivo de personas con parálisis cerebral ha expresado la desacreditación que supone la parálisis cerebral en su vida diaria y concretamente en la gestión de sus relaciones con la administración. Expresan esta desacreditación en la falta de confianza de su entorno más cercano a la hora de acometer tareas propias del mundo adulto y sienten como algo negativo el estar permanentemente acompañados y supervisados.

- "(en la consulta del médico) Prefiero entrar sólo, mi padre se mete en la conversación"

- "Sólo puedo entrar sin mi padre si entra conmigo mi hermana"

El estigma expresado por las personas con parálisis cerebral participantes en la investigación no se limita a sus capacidades funcionales. Aunque estas son el origen del mismo, terminan generando una desacreditación de la capacidad de la persona. Se produce un efecto de traspaso del estigma desde la funcionalidad a la capacidad, siendo este un fenómeno propio del conflicto con el cuerpo normativo: si no puedo ir sólo caminando hasta la consulta del médico, no puedo gestionar sólo mis relaciones con el médico.

La presencia del estigma no concluye en la desacreditación de toda la capacidad, sino que se extiende a toda persona. Las personas con parálisis cerebral han relatado como se produce en su entorno más cercano un proceso mediante el cual, al relacionarse a través del estigma en diferentes espacios, terminan siendo desacreditados a priori en todas partes. Esto produce el efecto de que sus limitaciones funcionales para desarrollar determinadas actividades, termina limitando su autonomía moral: si no puedo ir sólo caminando hasta la consulta del médico, no puedo gestionar sólo mis relaciones con el médico, y por tanto no puedo gestionar autónomamente mi salud.

Esta presencia del estigma explica las dificultades encontradas en las pruebas piloto relacionadas con la comprensión. En los datos recogidos por el cuestionario ACDM-16 se encontró que las personas con parálisis cerebral no tenían limitaciones funcionales para poder asumir la responsabilidad que implica la autonomía moral de gestionar sus relaciones con la administración. Sin embargo, relatan sus experiencias y expectativas como si fuese así, ya que han visto mermada su capacidad de decisión al extenderse el estigma a toda la persona. Por este motivo, mostraron dificultades a la hora de comprender las gestiones que realizaban, ya que formaban parte de la gestión de una vida adulta que se les impide gestionar off-line y que desconocen.

La barrera no se localiza por tanto en el diseño, sino en la socialización de la persona. La persona termina autolimitando sus capacidades en función de la respuesta que recibe de la sociedad, mostrando un caso paradigmático de autoestigma. Esto se traduce en resultados en las pruebas de accesibilidad que no son comprensibles desde el paradigma técnico-funcional y que exigen un análisis sociológico.

El colectivo de personas con lesión medular se ha revelado más diverso y heterogéneo. En primer lugar, no es posible hablar de una identidad colectiva tan definida, debido a que no existe una presencia tan marcada del estigma. Al ser esta una discapacidad sobrevenida, las personas con lesión medular provienen de socializaciones previas diversas. Es cierto que al compartir una experiencia tan trascendente como la lesión, se produce una identificación colectiva, pero esta no ha sido expresada con tanto consenso como en el caso del colectivo de parálisis cerebral. Se puede afirmar que la lesión medular es un colectivo donde, dentro de unas series de elementos comunes, se refleja el resto de la sociedad, mientras que la parálisis cerebral es un colectivo con identidad propia.

La diferencia de este colectivo en la vivencia del estigma resultó relevante para comprender los resultados de las pruebas de accesibilidad a la administración pública. Si en el caso del colectivo con parálisis cerebral encontrábamos autolimitaciones en la autonomía moral que impedían ejercer la autonomía funcional, en el colectivo con lesión medular no encontramos esta barrera. Al deliberar sobre los resultados obtenidos en las pruebas de accesibilidad, las barreras funcionales aparecen en primer plano. Haber vivido la socialización primaria desde fuera de la discapacidad parece ser un elemento explicativo de la autonomía del colectivo en la gestión de su vida adulta, incluyendo las relaciones con la administración, a pesar de las barreras y dificultades experimentadas. En este caso, el análisis sociológico se reveló de menor utilidad que en el caso del análisis de resultados del colectivo con parálisis cerebral. 
Como se ha reflejado previamente, el estigma ha mostrado una presencia distinta en ambos colectivos, estando más presente en el colectivo donde la socialización primaria se ha vivido dentro de la discapacidad. Sin embargo, además de las diferencias derivadas de la mayor o menor presencia del estigma, este se ha mostrado de manera diferente. En el caso del colectivo de personas con parálisis cerebral se ha encontrado un estigma de naturaleza ambivalente y en el caso del colectivo de lesión medular se ha encontrado un estigma bidireccional. Las diferencias responden a las diferentes posiciones de poder percibidas por ambos colectivos con respecto al resto de la sociedad.

Para que exista un estigma, debe existir una relación de poder desigual que establezca quien es el estigmatizado y quien el estigmatizador. En el caso de las personas con parálisis cerebral, el desequilibrio de poder en su contra es claro y el estigma es más fuerte entre su colectivo. Sin embargo, en el colectivo de personas con lesión medular encontramos discursos propios de una relación que, si no es de igual a igual, pretende serlo. Las personas con lesión medular sienten el estigma y así lo han manifestado en los grupos focales. Pero también han reproducido discursos donde ellos estigmatizan a la sociedad.

○ "El mundo no está diseñado para la discapacidad"

-(refiriéndose al diseño del entorno) ¿Quiénes son los normales? Antes éramos subnormales, luego anormales, ahora....."

Este tipo de sentencias muestra un colectivo que se siente en una posición de poder suficiente como para responder a la estigmatización que sufre. La socialización primaria vivida fuera de la discapacidad y dentro del cuerpo normativo les permite hablar de igual a igual a la sociedad que ahora les estigmatiza. Esta es una circunstancia que la particular socialización primaria del colectivo de personas con parálisis cerebral les impide hacer.

Entre las personas con parálisis cerebral se han encontrado discursos que muestran que el estigma, a pesar de contar con una fuerte presencia, muestra ambivalencias. Por ejemplo, en el caso de las visitas médicas dentro y fuera de la asociación para la discapacidad APAM donde tuvieron lugar tanto las pruebas de accesibilidad como los grupos focales. Cuando las visitas médicas son en los Centros de Salud Públicos en compañía de la familia, reproducen problemas funcionales que les impiden realizar estas gestiones de manera autónoma.

○ "Si no hubiese escalones entraría yo sola y estaría más tranquila"

○ "Entra mi madre porque yo no me entero"

Sin embargo, cuando las visitas médicas son dentro de la asociación APAM y sin la compañía de familiares, estos problemas desaparecen y las mismas barreras son superadas. Los individuos son capaces de realizar en la asociación tareas de las que no son capaces fuera, a pesar de partir de una situación con las mismas capacidades funcionales. Es el autoestigma quien construye las barreras con los mismos elementos de su cuerpo y del entorno. En otras palabras, el estigma tiene mayor o menor presencia en función del contexto en que desenvuelva el individuo. Si la familia -elemento de la socialización primaria- está presente y el espacio es un centro público -vida adulta-, el autoestigma aparece con mayor influencia. Pero si la familia no está presente y el espacio es cercano -asociación para la discapacidad-el autoestigma se diluye.

O "APAM es nuestro espacio, podemos hacer cosas"

○ "Aquí sí, en casa un 0 a la izquierda"

Es razonable pensar que esta ambivalencia del estigma influye igualmente a la hora de ejecutar determinadas acciones a través de la administración electrónica.

\section{CONCLUSIONES}

La principal conclusión que se ha extraído de la investigación es que las experiencias previas a las pruebas influyen decisivamente en el desarrollo de las mismas. Por tanto, no se pueden estudiar de forma aislada y se hace imprescindible la comprensión del contexto de los usuarios para comprender los resultados de las pruebas de accesibilidad. En el caso de los análisis de accesibilidad realizados en esta investigación, las pruebas de acceso a la administración pública electrónica corresponden a espacios de gestión de la vida adulta. Estas circunstancias superan la perspectiva del paradigma técnicofuncional y sólo son comprensibles mediante un análisis sociológico.

La socialización primaria dentro o fuera de la discapacidad es el elemento sociológico que se ha mostrado más relevante para la comprensión del contexto y su influencia en los resultados de la pruebas de accesibilidad. El colectivo de personas con parálisis cerebral, socializado desde su nacimiento en la discapacidad, muestra una fuerte homogeneidad en torno a la presencia del estigma y el autoestigma. Debido a esto, los individuos de dicho colectivo muestran en las pruebas de accesibilidad autolimitaciones en sus capacidades de gestión de la vida adulta que no están justificadas por sus capacidades funcionales. En consecuencia, las soluciones de accesibilidad y/o diseño universal no son suficientes para permitir su acceso a la administración en igualdad de condiciones que el resto de ciudadanos. En el caso del colectivo de personas con lesión medular la presencia del estigma es menor y se presenta de forma más heterogénea. La socialización primaria vivida fuera de la discapacidad es igual o más relevante a la hora de explicar la interacción de los individuos con la administración electrónica. Por supuesto, existen elementos comunes al compartir todos los individuos la vivencia de la lesión, pero entre las barreras destacadas, priman las barreras funcionales dependientes de la accesibilidad del diseño. En este caso, el paradigma de diseño universal es válido para explicar y comprender la mayor parte de las barreras.

Estas particularidades que diferencian de forma tan relevante a dos colectivos en principio no tan diferentes desde el punto de vista técnico-funcional, nos permite afirmar que las políticas públicas destinadas a la aplicación de la Ley 11/2007 LAECSP (BOE, 2007) 
deben incluir acciones de intervención social en estos colectivos, además de trabajos sobre la tecnología. Es importante que el sector público incorpore en sus regulaciones e intervenciones la perspectiva sociológica para solucionar problemas relacionados con la accesibilidad a la tecnología, tanto a la administración electrónica en particular, como el acceso a la sociedad de la información en general.

Estos resultados y conclusiones ofrecen una respuesta afirmativa a la pregunta de investigación planteada sobre la existencia de elementos sociales, propios del estudio por parte de la ciencia sociológica, que se revelen fundamentales a la hora de estudiar la accesibilidad a la tecnología por parte de las personas con discapacidad. A lo largo de la investigación han surgido diferentes elementos sociológicos fundamentales para interpretar los datos recogidos.

Por su parte, la hipótesis de investigación fue definida como: "entre individuos de funcionalidades similares y con el uso de los mismos habilitadores tecnológicos, encontraremos diferencias en sus capacidades derivadas de sus diferencias de socialización". Esta hipótesis respondía a nuestro objetivo principal de ofrecer información científicamente relevante sobre la importancia de la sociología para el estudio y comprensión de la accesibilidad a la tecnología. Es posible afirmar que la hipótesis se ha confirmado y el objetivo se ha cumplido.

El cumplimiento de este objetivo tiene como consecuencia la necesidad de incluir la sociología en los diseños de análisis de accesibilidad. Este planteamiento supera los paradigmas actuales que plantean únicamente los niveles de análisis heurístico por expertos, análisis con usuarios o perspectivas multidisciplinares.(Nielsen, 1994; 2001; Hassan, 2003; 2004; Granollers y Saltiveri, 2009; Valero, 2011). Los resultados de esta investigación reivindican mayor profundidad sociológica en el análisis de las experiencias de los potenciales usuarios en su relación con la tecnología que se evalúa. Esta inclusión de la sociología como perspectiva necesaria para el estudio de la accesibilidad no debe comprenderse como una mera agregación de nuevos conocimientos procedentes de una ciencia complementaria a las anteriores, sino que invita a un cambio de planteamiento desde un paradigma centrado en el sujeto hasta otro que supere el individualismo conceptual y permita acercarse al fenómeno de la accesibilidad desde una observación más amplia que incluya identidades colectivas, procesos de socialización, etc. Igualmente, no sólo deben transformarse los estudios de accesibilidad, sino también la misma sociología, que precisaría un mayor protagonismo del paradigma sociotécnico, donde se comprende la tecnología como un hecho social y no como un hecho neutro propio de otras áreas de conocimiento (Bloor, 1991; Domènech y Tirado, 1998; Law y Hassard, 1999; Blanco, 2002; Latour 2005; Sádaba y Gordo, 2008; SánchezCriado, 2008).

En resumen, los resultados de nuestra investigación nos permiten reivindicar la sociología como ciencia útil y necesaria para los estudios de accesibilidad. En relación con el elemento particular del acceso a la administración electrónica, el cumplimiento de normativas y leyes como la Ley 11/2007 de Acceso Electrónico de los Ciudadanos a los Servicios Públicos (BOE, 2007) que establece la obligación de que los ciudadanos españoles puedan realizar todas sus gestiones con la administración pública de forma electrónica en igualdad de condiciones de acceso a los servicios, sólo puede alcanzarse incluyendo el trabajo sociológico y la intervencíón social entre las políticas públicas relacionadas. La intervención meramente técnica responde a un paradigma técnico-funcional, que si bien es útil en parte, está fundamentado en la complementación entre el paradigma biopsicosocial y el diseño universal, o, en otras palabras, en el encaje entre funcionalidades y tecnologías. Este paradigma resulta insuficiente para comprender todos los procesos que pueden observarse en un estudio de accesibilidad del tipo que hemos descrito en este artículo. Se hace imprescindible la inclusión de la sociología como ciencia relevante en la definición de las políticas públicas de las diferentes administraciones para conseguir la accesibilidad a las nuevas tecnologías por parte de personas con discapacidad.

Por último, cabe destacar que los resultados de nuestra investigación que nos permiten realizar estas afirmaciones se refieren únicamente al peso de un concepto como el autoestigma en la relación/interacción de la persona con discapacidad con la tecnología. Se pueden plantear múltiples propuestas de investigación futura que fundamenten nuestras afirmaciones, como estudios basados en el género, el nivel educativo, la clase social, el estatus, etc. en sus diferentes combinaciones y/o coexistencias con la discapacidad y su influencia en el acceso a la sociedad de la información a través de tecnologías de diseño normativo. Desde aquí hacemos una llamada a la sociología para abordar este espacio de investigación que se abre.

\section{REFERENCIAS}

Aretio, A. (2010). "Una mirada social al estigma de la enfermedad mental". Cuadernos de Trabajo Social , 23: 289-300.

Barton, L. (1998). "Sociología y discapacidad: algunos temas nuevos”. Pp 19-33 en Discapacidad y sociedad. Madrid: Ediciones Morata.

BOE. (2006). "Ley 39/2006, de 14 de diciembre, de Promoción de la Autonomía Personal y Atención a las personas en situación de dependencia" Boletín Oficial del Estado. 299: 44142-44156, 15 de diciembre de 2006.

Coller, X. (2000). Estudios de casos. Madrid: CIS.

Conde, R., Aguilar, M., (2012). "ACDM-16: Propuesta de cuestionario para evaluar la accesibilidad de los servicios de administración pública electrónica en España para las personas con discapacidad motora." Presentado en VI Congreso Andaluz de Sociología. La constitución de las sociedades. Cádiz.: Asociación Andaluza de Sociología.

Conde, R. (2014). Evolución del concepto de discapacidad en la sociedad contemporánea: de cuerpos enfermos a sociedades excluyentes. Praxis sociológica, 18: 155-175.

Díaz Velázquez, E. (2010). "Ciudadanía, identidad y exclusión social de las personas con discapacidad". Política y Sociedad , 47 (1): 115-135.

Dominguez, M. D. (2008). "La práctica conversacional del grupo de discusión: jóvenes, ciudadanía y nuevos derechos". Pp 98-125 en Estrategias y prácticas cualitativas de investigación social. Madrid: Pearson.

Etxeberría, X. (2008). La condición de ciudadanía de las personas con discapacidad intelectual. Bilbao: Universidad de Deusto.

Ferrante, C. F. (2010). "El habitus de la discapacidad: la experiencia corporal de la dominación en un contexto económico periférico". Política y Sociedad 47 (1): 85-104

Ferreira, M. R. (2006). "Sociología de la discapacidad: una propuesta teórica crítica”. Nomadas. Revista Crítica de Ciencias Sociales y Jurídicas. , 1 (13).

Ferreira, M. A. (2008). "Una aproximación sociológica a la discapacidad desde el modelo social: apuntes caracteriológicos". REIS 124: 141-174.

Ferreira, M. A. (2009). "Discapacidad, exclusión social y tecnologías". Política y Sociedad 46 (1 y 2): 237-253. 
Ferreira, M. (2010). "De la minusvalía a la diversidad funcional: un nuevo marco teórico-metodológico". Política y Sociedad , 47 (1): 45-65.

Giner, S., Lamo de Espinos, E., Torres, C., (1998). Diccionario de Sociología. Madrid: Alianza.

Goffman, E. (1961). Asylums: Essays on the Social Situation of Mental Patients and Other Inmates. Anchor Books.

Goffman, E. (1963). Stigma: the Management of Spoiled Identity. Harmondsworth: Penguin.

Goffman, E. (1971). Relations in Public: Microstudies of the Social Order. New York: Basic Books.

Goffman, E. (1981). "A reply to Denzin and Keller". Contemporary Sociology: A Journal of Reviews 10: 60-68.

Goffman, E. (1997). La presentación de la persona en la vida cotidiana. Buenos Aires: Amorrortu.

Granollers i Saltiveri, T. (2007). "MPlu+a. Una metodología que integra la ingeniería del software, la interacción persona-ordenador y la accesibilidad en el contexto de equipos de desarrollo multidisciplinares". Tesis Doctoral . Lleida: Universitat de Lleida. Departament d'Informàtica i Enginyeria Industrial.

Greebaum, T. L. (1998). The Handbook for focus Group Research. . Thousand Oaks.

Hassan, Y. M. (2003). "Qué es la Accesibilidad Web". No solo usabilidad: revista multidisciplinar sobre personas, diseño y tecnología.
http://www.nosolousabilidad.com/articulos/accesibilidad.htm

Hassan, Y. M. (2004). "Propuesta de adaptación de la metodología de diseño centrado en el usuario para el desarrollo de sitios web accesibles". Revista Española de Documentación Científica , 3 (27): 330-344.

Henry, S. L. (2001). "Adapting the Design Process to Adress more Customers in more Situations". Presented at UPA (Usability Professionals' Association) 2001 Conference.

Krueger, R. A. (1994). Focus Group. A Practical guide for Applied Research . London: Thousand Oaks.

Maraña, J. L. (2003). "El movimiento de vida independiente en España". Pp 259281 En El Movimiento de Vida Independiente: experiencias internacionales. Madrid: Fundación Luis Vives.

Marrero-Guillamón, I. (2012). "Descentrar el sujeto. Erving Goffman y la teorización del sujeto". Revista Internacional de Sociología RIS , 70 (2): 311-326.

Mead, G. (1993). Espíritu, persona y sociedad: desde el punto de vista del conductivismo social. México/ Buenos Aires/ Madrid: Paidós.

Nielsen, J. M. (1994). Usability Inspection Methods. New York: Wiley \& Sons.
Nielsen, J. (2001). Beyond Accessibility: Treating People with Disabilities as People. New York: Alertbox.

ONU. (2006). "Convención sobre los derechos de las personas con discapacidad y su Protocolo Facultativo". Consulta 06 de febrero de 2013 (http.//www.un.org/disabilities/documents/convention/convoptprot-s.pdf)

Palacios, A. R. (2006). El modelo de la diversidad. La bioética y los derechos humanos como herramienta para alcanzar la plena dignidad en la diversidad funcional. Madrid: Diversitas.

Palacios, A. R. (2007). El modelo de la diversidad. Madrid: Diversitas.

Rogero, J. (2010). Los tiempos del cuidado. El impacto de la dependencia de los mayores en la vida cotidiana de sus cuidadores. (Colección Estudios. Serie Dependencia. ed.). Madrid: IMSERSO.

Romañach, J. L. (2005). Diversidad funcional, nuevo término para la lucha por la dignidad en la diversidad del ser humano. Madrid: Foro de Vida Independiente.

Sádaba, I., \& Gordo, A. (2008). "La tecnología es política por otros medios" Pp 9-22 en . Cultura digital y movimientos sociales. Madrid: Catarata.

Scambler, G. (2009). "Health-related stigma". Sociology of Health and IIIness, 31 (3): 441-455.

Toboso, M. G. (2010). "Cuerpos, capacidades, exigencias funcionales... y otros lechos de Procusto". Política y Sociedad , 47 (1): 67-83.

Valero, M. V. (2011). Investigación sobre las tecnologías de la sociedad de la información para todos. Madrid: CENTAC.

Vallés, M. S. (2003). Técnicas cualitativas de investigación social. Reflexión metodológica y práctica profesional. Madrid: Síntesis.

Van Campenhoudt, L. C. (2005). La méthode d'analyse en groupe. Applications aux ph'enomènes sociaux. . Paris: Dunod.

Vanderheiden, G. (2000). Fundamental Principles and Priority Setting for Universal Usability. Proceedings of Conference on Universal Usability (CUU) Association for Computing Machinery.

World Health Organitation. (1980). International Classification of Impairments, Disabilities and Handicaps. WHO Library Cataloguing-in-Publication Data.

World Health Organitation. (2001). International Classification of Functioning, Disability and Health. Geneve: WHO Library Cataloguing-in-Publication Data.

World Health Organitation. (2002). Towards a Common Language for Functioning, Disability and Health:ICF. (T. p. textuales, Trad.) Geneva: World Health Organization.

Yin, R. (2009). Case study research: design and methods. California: Thousand Oaks. 